\title{
Malária no Estado de São Paulo: Avaliação de Aspectos da Vigilância Epidemiológica *
}

\author{
Dalva Marli Valério Wanderley \\ José Carlos Rehder de Andrade \\ Maria José Chinelatto $P$. Alves \\ Maria Cecília Goi Porto Alves \\ Marina Ruiz de Mattos \\ Sérgio de Mello Gurgel \\ Ricardo Pereira Igreja **
}

São apresentadas informações epidemiológicas da malária no Estado de São Paulo referentes aos anos de 1985 e 1986.

Selecionou-se, através das fichas de investigação epidemiológica, um indicador para a avaliaçấo do tempo decorrido entre o início dos primeiros sintomas/data da coleta da lâmina e início do tratamento, que foi agrupado segundo categorias denominadas tipo de procura.

Foram consideradas nesta avaliação as seguintes variáveis: a espécie de plasmódio diagnosticada, a existência de passado malárico e a utilização de serviços de saúde anteriores à confirmação do diagnóstico pela SUCEN.

Os autores concluem que entre as variáveis estudadas a existêncic de malária anterior se constitui no principal fator explicativo para os diferentes tempos decorridos entre os primeiros sintomas e comprovação diagnóstica.

\section{UNITERMOS: Malária, Epidemiologia}

\section{INT RODUÇÃO}

* Trabalho apresentado no $X$ Congresso da Sociedade Brasileira de Parasitologia, Satvador, agosto de 1987.

* da Superintendência de Controle de Endemias (SUCEN), Rua Paula Souza, 166, CEP. O!C27. Säo Paulo.
Nos últimos anos o número de casos de malária diagnosticados no Estado de São Paulo tem sido ampliado (6), como reflexo direto da expansão da endemia no Brasil (2). O aumento de risco de instalação de focos da doença determinou a intensificação das ações de vigilância desenvolvidas pela Superintendência de Controle de Endemias (SUCEN). Ao mesmo tempo em que se buscava uma maior participação da rede básica prestadora de assistência médica à população, foi incrementada a vigilância nas áreas de acesso rodoferroviário do Estado, através do aumento da busca ativa de casos e de campanhas de divulgação, junto aos grupos de risco identificados (1).

Do ponto de vista epidemiológico, o período de parasitemia circulante nos pacientes é fator determi-

Cadernos de Saúde Pública, RJ, 5(3): 296-304, jul/set, 1989 
nante da possibilidade de infecção de anofelinos e conseqüentemente da variação do nível de transmissão. Neste sentido, o tempo decorrido entre o aparecimento dos sintomas de malária e a busca do diagnóstico, com posterior instituição de terapêutica apropriada, se constitui num importante parâmetro da vigilância da doença. O presente estudo tem como proposta avaliar este parâmetro, com base na notificação de casos de malária no Estado de São Paulo, nos anos de 1985 e 1986.

\section{MATERIAL E MÉTODOS}

A partir das fichas de investigação epidemiológica de casos de malária hemoscopicamente confirmados, selecionou-se os intervalos, em dias, entre a data do início dos primeiros sintomas e a data da coleta da lâmina. Tais intervalos variaram de zero a dez dias e mais, e serviram de referência para a tabulação das seguintes informaçōes: espécie de plasmódio diagnosticada, existência de passado malárico e utilização de serviços de saúde anteriores à confirmação do diagnóstico. $\mathrm{Na}$ quase totalidade dos casos, a data da coleta da lâmina coincidiu com a do início do tratamento. Assim, considerou-se iguais os intervalos entre primeiros sintomas e coleta da lâmina e primeiros sintomas e início do tratamento.

Os procedimentos adotados pela SUCEN frente a um caso de malária foram descritos em trabalho anterior (7).

Os 2.201 casos de malária, notificados em 1985 , foram processados pela Companhia de Processamento de Dados do Estado de São Paulo (PRODESP). As diferenças observadas nas totalizações obtidas são devidas a cruzamentos de itens cuja resposta numérica encontrava-se em branco ou preenchida com mais de uma altemativa.

Em 1986, das 2.686 notificações, foram recuperados 2.615 casos, utilizando-se tabulação manual, onde foram excluídos aqueles protocolos sem resposta e/ou com mais de uma alternativa em algum item. Não foram considerados 2 casos de infecção por Plasmodium malariae, bem como um caso que referia passado malárico desta mesma espécie de plasmódioo.

$\mathrm{Na}$ interpretação de resultados utilizou-se de testes de significância: paramétricos ( $t$-Student) e não paramétricos (Qui-Quadrado).

No sentido de se conhecer a distribuição dos pacientes, segundo o tempo entre os primeiros sintomas e coleta da lâmina, foram definidos alguns intervalos que pudessem resultar em categorias de importância epidemiológica. Desta forma, foram definidas as se- 
guintes categorias aqui denominadas tipo de procura: procura imediata (intervalo zero entre sintoma e coleta), procura precoce (representada pelos intervalos de 1 a 3 dias), procura intermediária (compreendida pelo período de 4 a 9 dias) e procura tardia (a partir do 10: dia).

Deve se considerar que a importância destes intervalos está relacionada nấo apenas a detecção precoce dos casos e seu tratamento, mas a diminuição da possibilidade de transmissão em áreas de risco, isto é, que contam com a presença do vetor. Aliado a este fato, é necessário ter em conta que se para a malária por $P$. falciparum o periodo de transmissibilidade está incluído no intervalo superior a 6 dias entre os primeiros sintomas e coleta da lâmina, para a malária por $P$. vivax o período de transmissibilidade antecede o intervalo zero, uma vez que a parasitemia sexuada é encontrada no sangue até 3 dias antes do aparecimento dos primeiros sintomas (3).

\section{RESULTADOS E COMENTÁRIOS}

A distribuição dos casos de malária notificados em 1985 e 1986, segundo o tipo de procura de diagnóstico pode ser evidenciada na Tabela 1.

TABELA 1

Tipo de procura de diagnóstico da malária no Estado de São Paulo, 1985 e 1986.

\begin{tabular}{l|rrr|rr}
\hline \multirow{2}{*}{$\begin{array}{l}\text { Tipo de procura } \\
\text { (em dias) }\end{array}$} & \multicolumn{2}{|c|}{1985} & \multicolumn{2}{c}{1986} \\
\cline { 2 - 5 } & \multicolumn{1}{|c|}{ No } & $\%$ & No & $\%$ \\
\hline Imediata (zero) & 149 & 6,9 & 241 & 9,2 \\
Precoce (1 a 3) & 1032 & 47,5 & 1275 & 48,8 \\
Intermediária (4 a 5) & 383 & 17,6 & 451 & 17,2 \\
(6 a 9) & 339 & 15,6 & 335 & 12,8 \\
Tardia (10 e +) & 268 & 12,4 & 313 & 12,0 \\
\hline Total & 2171 & 100,0 & 2615 & 100,0 \\
\hline
\end{tabular}


Observa-se diferença significativa ( $\mathrm{p} 0,01)$ no padrão de frequência dos dois anos analisados, possivelmente ocasionada por mudanças ocorricias para os tipos de procura imediata e intermediária de 6 a 9 dias. Ressalta-se que apesar dessa mudança apontar para uma melhora nos tempos observados (acréscimo de $33,3 \%$ na procura imediata e decréscimo de $18,0 \%$ no intervalo de 6 a 9 dias), o resultado para os que tiveram uma procura tardia manteve-se no mesmo patamar.

A procura imediata, por significar acesso a diagnóstico no mesmo dia em que apareceram os primeiros sintomas, deve se referir a pessoas que, além de conhecerem de antemão esses sint omas, sabiam também do atendimento oferecido pela SUCEN. Apesar da grande maioria dos pacientes contrair a doença fora do Estado $(93,8$ e $90,5 \%$ dos casos notificados em 1985 e 1986 foram importados $(4,5))$, supõe-se que pessoas incluídas no primeiro intervalo já se encontravam em municípios paulistas quando do surgimento da sintomatologia.

As mesmas consideraçōes podem ser feitas em relação aos pacientes incluídos no intervalo de tempo equivalente à procura precoce.

Pode-se observar ainda (Tabela 1) que nos dois primeiros intervalos incluem-se mais da medade dos pacientes.

A classificação em procura intermediária está associada a uma demora na busca do serviço, que pode interferir significativamente no prognóstico do paciente e no aumento da possibilidade de transmissão da doença. Nesse sentido pode-se considerar elevada a porcentagem de pacientes classificados no intervalo, uma vez que cerca de um terço deles estão aí incluídos. Deve ser ressaltado que nos 11 óbitos notificados em 1986 o tempo médio entre os primeiros sintomas e o diagnóstico pela SUCEN foi de 8 dias (5).

Com referência a procura tardia, acentua-se a questão da gravidade em relação ao prognóstico, principalmente casos de malária falcípara, e de aumento da possibilidade de transmissão, também já assinalada para o período anterior. Dessa forma, os valores observados para este intervalo, embora pequenos, se comparados aos restantes, assumem grande importância quando analisadas as conseqüências aí implicadas.

Salienta-se que para 1986 , onde o levantamento permitiu a visualização do final desse intervalo, $1,2 \%$ dos casos apresentaram procura superior a 30 dias.

Uma vez colocada a questão da gravidade em relação ao tipo de procura intermediária e tardia e os diferentes tipos de malária, torna-se importante a avaliação desta associação (Tabela 2). 
TABELA 2

Tipo de procura de diagnóstico de malária segundo a espécie de plasmódio diagnosticada,

no Estado de São Paulo, 1985 e 1986.

\begin{tabular}{|c|c|c|c|c|c|c|c|c|}
\hline \multirow{3}{*}{$\begin{array}{l}\text { Tipo de procura } \\
\text { (em dias) }\end{array}$} & \multicolumn{4}{|c|}{1985} & \multicolumn{4}{|c|}{1986} \\
\hline & \multicolumn{2}{|c|}{ P. vivax } & \multicolumn{2}{|c|}{ P.falcipenm } & \multicolumn{2}{|c|}{ P.vivax } & \multicolumn{2}{|c|}{$\underline{\text { P. falcipanm }}$} \\
\hline & $n \stackrel{2}{2}$ & $\%$ & $\mathrm{n} \cong$ & $\%$ & 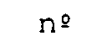 & $\%$ & $n \cong$ & $\%$ \\
\hline Imediata (zero) & 75 & 5.7 & 53 & 8,9 & 157 & 9,4 & 67 & 8,4 \\
\hline Precoce ( 1 a 3$)$ & 598 & 45,7 & 291 & 48,9 & 807 & 48,0 & 407 & 51,0 \\
\hline Intermediária (4 a 5) & 260 & 19,9 & 92 & 15,5 & 294 & 17,5 & 133 & 16,7 \\
\hline$(6$ a 9$)$ & 223 & 17,0 & 80 & 13,4 & 229 & 13,6 & 95 & 11,9 \\
\hline Tardia $(10 \mathrm{e}+)$ & 153 & 11,7 & 79 & 13,3 & 193 & 11,5 & 96 & 12,0 \\
\hline$\overline{\text { Total }}$ & 1309 & 100,0 & 595 & 100,0 & 1680 & 100,0 & 798 & 100,0 \\
\hline
\end{tabular}

FONTE : SUCEN

Quando se analisa o ano de 1985 observa-se que existem diferenças nos tipos de procura de diagnóstico de malária por $P$. vivax e $P$. falciparum ( $\mathrm{p}<0.01$ ), indicando uma melhor situação para este último, exceção feita a procura tardia, justamente onde as consequiências da malária por $P$. falciparum ficam agravadas.

O quadro para $P$. vivax altera-se em 1986 $(p<0.001)$, havendo indicação de melhora nos tempos de procura imediata e precoce e, tornando-se então, semelhante ao apresentado pela malária por $P$. falciparum $(p>0.30)$. Este fato é de grande importância, na medida em que pacientes com $P$. vivax se constituem fontes de infecção para os anofelinos desde o início da sintomatologia. Assim, o rápido diagnóstico e simultânea instituição da terapêutica apropriada, além da recuperação do paciente, minimiza o risco de transmissão.

O pressuposto de que os tipos de procura pudessem estar relacionados ao fato do paciente já ter tido malária anteriormente, levou ao exame da distribuição dos casos segundo seu passado malárico (Tabela 3 ). 
TABELA 3

Tipo de procura de diagnóstico de malária segundo a existência ou não de passado malárico, no Estado de Săo Paulo, 1985 e 1986.

\begin{tabular}{|c|c|c|c|c|c|c|c|c|}
\hline $\begin{array}{l}\text { Tipo de procura } \\
\text { (em dias) }\end{array}$ & \multicolumn{4}{|c|}{$\begin{array}{c}1985 \\
\text { Passado malárico } \\
\text { sim nao }\end{array}$} & \multicolumn{4}{|c|}{$\begin{array}{c}1986 \\
\substack{\text { Passado mal áric co } \\
\text { sim }} \\
\text { não }\end{array}$} \\
\hline Imediata (zero) & 127 & 8,7 & 22 & 3,1 & 193 & 10,5 & 48 & 6,2 \\
\hline Precoce (1 a 3$)$ & 772 & 53,2 & 260 & 36,2 & 1024 & 55,5 & 251 & 32,6 \\
\hline Intermediária (4 a 5) & 218 & 15,0 & 165 & 22,9 & 306 & 16,6 & 145 & 18,8 \\
\hline$\left(\begin{array}{lll}6 & \text { a } & 9\end{array}\right)$ & 186 & 12,8 & 153 & 21,3 & 160 & 8,7 & 175 & 22,8 \\
\hline Tardia $(10 \mathrm{e}+)$ & 149 & 10,3 & 119 & 16,5 & 163 & 8,7 & 150 & 19,6 \\
\hline Total & $145 \dot{2}$ & 100,0 & 719 & 100,0 & 1846 & 100,0 & 769 & 100,0 \\
\hline
\end{tabular}

FONIE: SUCEN

Analisando os dados da Tabela 3 nota-se que a diferença de comportamento das freqüências observadas nos 2 grupos em estudo é significante $(p<0.001)$. Os pacientes que referem passado malárico obtêm diagnóstico muito mais rapidamente que aqueles primoinfectados, isto é, mais da metade dos casos com malária anterior procuram diagnóstico imediatamente ou precocemente, sendo inversa a situação dos que ficam doentes pela primeira vez.

Este fato reforça a afirmação feita anteriormente de que nos intervalos de tempo correspondentes a procura imediata e precoce incluem-se, na sua maioria, pacientes que já conheciam o serviço prestado pela SUCEN, os sintomas da malária, uma vez que já a tinham contraído.

As porcentagens de pacientes com passado malárico são de $66,9 \%$ e $70,6 \%$ para os anos de 1985 e 1986 .

A associação entre os tipos de procura e a utilização de serviços médicos anteriores à SUCEN pode ser evidenciada na Tabela 4.

Cadernos de Saúde Pública, RJ, 5(3): 296-304, jul/set, 1989 
TABE LA 4

Típo de procura de diagnóstico de malária segundo a utilizaçāo de serviços médicos anteriores à SUCEN. Esta do de São Paulo, 1985 e 1986.

\begin{tabular}{|c|c|c|c|c|c|c|c|c|}
\hline \multirow[t]{2}{*}{$\begin{array}{l}\text { Tipo de procura } \\
\text { (em dias) }\end{array}$} & \multicolumn{4}{|c|}{\begin{tabular}{c}
\multicolumn{2}{c}{1985} \\
Serviço médico anterior \\
Sim
\end{tabular}} & \multicolumn{4}{|c|}{\begin{tabular}{|cc} 
& 1986 \\
Serviço médico anterior \\
Sim & Não
\end{tabular}} \\
\hline & $n=$ & $\%$ & 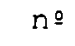 & $\%$ & $n \varrho$ & $\%$ & $n \cong$ & $\%$ \\
\hline Imediata (zero) & 56 & 4,3 & 92 & 10,1 & 106 & 7,4 & 135 & 11,5 \\
\hline Precoce $\left(\begin{array}{lll}1 & \text { a } & 3\end{array}\right)$ & 530 & 40,2 & 519 & 56,7 & 562 & 39,1 & 713 & 60,5 \\
\hline Intermediária ( 4 a 5 ) & 275 & 20,9 & 129 & 14,1 & 295 & 20,5 & 456 & 13,3 \\
\hline (6 a 9 ) & 250 & 19,9 & 99 & 10,8 & 239 & 16,5 & 96 & 8,2 \\
\hline Tardia $(10 \mathrm{e}+)$ & 206 & 15,6 & 76 & 8,3 & 237 & 16,5 & 76 & 6,5 \\
\hline Total & 1317 & 100,0 & 915 & 100,0 & 1439 & 100,0 & 1176 & 100,0 \\
\hline
\end{tabular}

FONWE: SUCEN

Observa-se que um grande percentual dos doentes de malária foi buscar na SUCEN, o primeiro atendimento $(41,0 \%)$ em 1985 e $45,0 \%$ em 1986), evidenciando mais uma vez o conhecimento existente em relação a este serviço.

O tempo médio de procura calculado para 1986 foi de 3,4 e 5,6 dias, respectivamente, para indivíduos que procuraram diretamente a SUCEN e aqueles com passagem por outros serviços. A diferença de dias observada entre os dois grupos $(p<0.001)$ pode ter implicações do ponto de vîsta do prognóstico da doença e das ações de controle.

Os intervalos médios maiores observados entre individuos que procuraram algum serviço de saúde anterior à SUCEN podem ter duas explicações: demora na suspeita da malária por parte do serviço que prestou atendimento ou demora na procura desse serviço por parte dos pacientes. Neste grupo devem estar incluídos indivíduos primoinfectados.

\section{CONCLUSŌES}

- Entre as variáveis estudadas a existência de malária anterior se constituiu no principal fator explicativo 
para os diferentes tempos decorridos entre os primeiros sintomas e comprovação diagnóstica.

- A comparação entre os dois anos estudados mostrou para 1986 uma maior proporção de indivíduos com diagnóstico realizado mais precocemente.

- O comportamento dos pacientes que tiveram tempo decorrido entre os primeiros sintom as e a comprovação diagnóstica de até 3 dias (procura imediata e precoce), se mostrou diferente em relação a todas as variáveis analisadas quando comparado com aqueles que procuraram o diagnóstico após 4 dias de sintomas (procura intermediária e tardia).

Epidemiological data from malaria in São Paulo State referring to 1985 and 1986 are presented. The authors selected from the epidemiological investigation card an information for the evaluation on the time since the begining of the first symptoms up to the date of the blood slide examination and the date of the begining of the treatment. These data were put together according to some categories named: "kind of search". It was considered in this evaluation some information as: the kind of malaria diagnosed, the existence of prior malaria and the use of another health care before the malaria confirmation by Superinten dência de Controle de Endemias (SUCEN).

The authors concluded that between the informations studied the existence of prior malaria is the main factor that influences on the different period of times from the begining of first symptoms and malaria diagnose.

\section{REFERÊNCIAS BIBLIOGRÁFICAS}

1. ANDRADE, J. C. R. de et alii. Vigilância epidemiológica da malária no Estado de São Paulo. 11 p. (Trabalho apresentado no 1: Congresso Brasileiro de Saúde Coletiva, Rio de Janeiro, 22 a 26.09.86).

2. SUPERINTENDENCIA DE CAMPANHAS DE SAÚDE PÚBLICA. SUCAM. Relatório anual. Brasilia, 1986.

3.PESSOA, S. B. \& MARTINS, A. V. Parasitologia médica. 11: ed. Rio de Janeiro, Editora Guanabara Koogan, 1982.

4. SUPERINTENDÊNCIA DE CONTROLE DE ENDEMIAS - SUCEN. Relatório de Avaliação Epidemiológica do Programa de Erradicação da Malária no Estado de São Paulo. 21 p. Sảo Paulo, 1985 (mimeo).

5. SUPERINTENDENCIA DE CONTROLE DE ENDEMIAS - SUCEN. Relatório de Avaliação Epidemiológica do Programa de Erradicação da Malária no Estado de Sāo Paulo. 25 p. São Paulo, 1986 (mimeo). 
6. SUPERINTENDÊNCIA DE CONTROLE DE ENDEMIAS - SUCEN. Programa de Controle da Malária no Estado de São Paulo. Situação Atual. 1987. (Relatório discutido na $V$ Reunión de Directores de los Servicios Nacionales de Erradicación de la Malaria en las Américas, Bogotá, Colômbia, 1 a 7 de junho de 1987). 13 p. (mimeo).

7. WANDER LEY, D. M. V. et alii. Malária no Estado de Sâo Paulo, Brasil, 1980 a 1983. Rev. Saúde públ., S. Paulo, 19: $28-36,1985$. 